Published in final edited form as:

Am J Obstet Gynecol. 2018 September ; 219(3): 296.e1-296.e8. doi:10.1016/j.ajog.2018.05.011.

\title{
Neonatal Outcomes of Elective Early Term Births after Demonstrated Fetal Lung Maturity
}

\author{
Dr Alan T.N. Tita, MD, PhD, \\ Departments of Obstetrics and Gynecology, Obstetrics and Gynecology, the University of \\ Alabama at Birmingham, Birmingham, Alabama \\ Dr Kathleen A Jablonski, PhD, \\ Departments of Obstetrics and Gynecology, the George Washington University Biostatistics \\ Center, Washington, DC \\ Dr Jennifer L. Bailit, MD, MPH, \\ Departments of Obstetrics and Gynecology, Case Western Reserve University-MetroHealth \\ Medical Center, Cleveland, Ohio \\ Dr William A. Grobman, MD, MBA, \\ Departments of Obstetrics and Gynecology, Northwestern University, Chicago, Illinois \\ Dr Ronald J. Wapner, MD, \\ Departments of Obstetrics and Gynecology, Columbia University, New York, New York \\ Dr Uma M. Reddy, MD, MPH, \\ Departments of Obstetrics and Gynecology, the Eunice Kennedy Shriver National Institute of \\ Child Health and Human Development, Bethesda, Maryland.
}

Dr Michael W. Varner, MD,

Departments of Obstetrics and Gynecology, the University of Utah Health Sciences Center, Salt Lake City, Utah

Dr John M. Thorp Jr., MD,

Departments of Obstetrics and Gynecology, the University of North Carolina at Chapel Hill, Chapel Hill, North Carolina

Dr Kenneth J. Leveno, MD,

Departments of Obstetrics and Gynecology, the University of Texas Southwestern Medical Center, Dallas, Texas

Dr Steve N. Caritis, MD,

Correspondence: Alan T.N. Tita MD, PhD, $17006^{\text {th }}$ Avenue South, Suite 10270, Birmingham, AL 35233-7333, Phone: 205-934-9616, Fax: 205-975-9858, atita@uab.edu.

Publisher's Disclaimer: This is a PDF file of an unedited manuscript that has been accepted for publication. As a service to our customers we are providing this early version of the manuscript. The manuscript will undergo copyediting, typesetting, and review of the resulting proof before it is published in its final form. Please note that during the production process errors may be discovered which could affect the content, and all legal disclaimers that apply to the journal pertain.

This study was presented in part at the $35^{\text {th }}$ Annual Meeting of the Society for Maternal-Fetal Medicine in San Diego, CA February 4-7, 2015.

Financial Disclosure

The authors did not report any potential conflicts of interest. 
Departments of Obstetrics and Gynecology, the University of Pittsburgh, Pittsburgh, Pennsylvania

Dr Jay D. lams, MD,

Departments of Obstetrics and Gynecology, The Ohio State University, Columbus, Ohio

Dr George Saade, MD,

Departments of Obstetrics and Gynecology, the University of Texas Medical Branch, Galveston, Texas

Dr Yoram Sorokin, MD,

Departments of Obstetrics and Gynecology, Wayne State University, Detroit, Michigan

Dr Dwight J. Rouse, MD,

Departments of Obstetrics and Gynecology, Brown University, Providence, Rhode Island

Dr Sean C. Blackwell, MD,

Departments of Obstetrics and Gynecology, the University of Texas Health Science Center at Houston-Children's Memorial Hermann Hospital, Houston, Texas

Dr Jorge E. Tolosa, MD, MSCE, and

Departments of Obstetrics and Gynecology, Oregon Health \& Science University, Portland, Oregon

for the Eunice Kennedy Shriver National Institute of Child Health and Human Development (NICHD) Maternal-Fetal Medicine Units (MFMU) Network ${ }^{\star}$

*Other members of the Eunice Kennedy Shriver National Institute of Child Health and Human

Development Maternal-Fetal Medicine Units Network are listed in the Appendix.

\section{Abstract}

Background: Studies of early term birth after demonstrated fetal lung maturity show that respiratory and other outcomes are worse with early term birth $\left(37^{0}-38^{6}\right)$ even after demonstrated fetal lung maturity when compared with full term birth $\left(39^{0}-40^{6}\right)$. However these studies included medically indicated births and are therefore potentially limited by confounding by the indication for delivery. Thus the increase in adverse outcomes might be due to the indication for early term birth rather than the early term birth itself.

Objective: We examined the prevalence and risks of adverse neonatal outcomes associated with early-term birth after confirmed fetal lung maturity as compared with full term birth in the absence of indications for early delivery.

Study Design: Secondary analysis of an observational study of births to 115,502 women in 25 hospitals in the United States from 2008-11. Singleton non-anomalous births at 37-40 weeks with no identifiable indication for delivery were included; early term births after positive fetal lung maturity testing were compared with full-term births. The primary outcome was a composite of death, ventilator for $\geq 2$ days, CPAP, proven sepsis, pneumonia or meningitis, treated hypoglycemia, hyperbilirubinemia (phototherapy) and 5 min Apgar < 7 . Logistic regression and propensity score matching (both 1:1 and 1:2) were used.

Results: 48,137 births met inclusion criteria; the prevalence of fetal lung maturity testing in the absence of medical or obstetric indications for early delivery was $0.52 \%(\mathrm{n}=249)$. There were 180 
$(0.37 \%)$ early-term births after confirmed pulmonary maturity and 47,957 full-term births. Women in the former group were more likely to be non-Hispanic White, smoke, have received antenatal steroids, have induction and have a cesarean. Risks of the composite (16.1\% vs. 5.4\%; adjusted odds ratio 3.2 ; $95 \%$ CI 2.1-4.8 from logistic regression) were more frequent with elective early term birth. Propensity scores matching confirmed the increased primary composite in elective early-term births: adjusted odds ratios 4.3 (1.8-10.5) for $1: 1$ and 3.5 (1.8-6.5) for 1:2 matching. Among components of the primary outcome, CPAP use and hyperbilirubinemia requiring phototherapy were significantly increased. Transient tachypnea of the newborn, neonatal intensive care unit admission and prolonged neonatal intensive care unit stay ( $>2$ days) were also increased with early term birth.

Conclusions: Even with confirmed pulmonary maturity, early term birth in the absence of medical or obstetric indications is associated with worse neonatal respiratory and hepatic outcomes compared with full-term birth, suggesting relative immaturity of these organ systems in early term births.

\section{Condensation}

Early-term birth after pulmonary maturity in the absence of indications for early delivery is associated with a higher risk of neonatal morbidity compared with full-term birth.

\section{Keywords}

Early-term births; elective delivery; fetal lung maturity testing; neonatal outcomes

\section{Introduction}

For neonates born in the preterm ( $<37$ weeks) or early term $\left(37^{0}-38^{6}\right.$ weeks) period, positive fetal lung maturity testing is associated with decreased risk for respiratory neonatal morbidity compared to gestational age matched cohorts delivered without testing.. ${ }^{1}$ Tests used for fetal lung maturity evaluate markers of pulmonary development such as lecithinsphingomyelin ratio, phosphatidylglycerol, fluorescence polariazation, and lamellar body counts. ${ }^{1}$ Several studies suggest that respiratory and other outcomes are worse with early term birth even after demonstrated fetal lung maturity when compared with full term ( $39^{0}$ $40^{6}$ weeks) birth, a period associated with a nadir in neonatal risks. ${ }^{2-5}$ As a result, the role of fetal lung maturity testing in determining early term and preterm births has been deemphasized. ${ }^{6}$ Once widely used and recommended by ACOG as an indication for delivery prior to 39 weeks, the use of fetal lung maturity has declined in recent years. ${ }^{7-8}$ However, the conclusions from studies that have compared outcomes of preterm or early-term births after fetal lung maturity testing to those of full-term births are limited by the inclusion of pregnancies with indications for early term birth such as hypertension and diabetes which are themselves associated with adverse pregnancy outcomes. Therefore, it is possible that the indication for the early term birth (i.e., confounding by indication) and not the gestational age may explain the association with worse outcomes. The potential for residual confounding by indication remains despite adjustment for the common indications. Few studies have examined the outcomes of early-term births with positive fetal lung maturity testing vs. full term births in pregnancies without medical or obstetric indication for early 
delivery. Therefore, we undertook this study to estimate the frequency and compare the risks of adverse neonatal outcomes with early term birth after positive fetal lung maturity testing compared to full term birth in the absence of indications for early delivery.

\section{Materials and Methods}

This is a secondary analysis of the Assessment of Perinatal Excellence (APEX) observational study of the Eunice Kennedy Shriver Maternal-Fetal Medicine Units Network. ${ }^{9}$ The APEX database includes detailed information on births to 115,502 women at 25 hospitals in the US from 2008-2011. The information was abstracted by chart review conducted by certified research staff at each center with IRB approval. The current study population included only singleton non-anomalous births at 37-40 weeks with no identifiable indication for early term delivery. Therefore, excluded were births to women with any medical or obstetrical indications for early delivery including: preeclampsia, eclampsia, gestational hypertension, or complicated chronic hypertension, oligohydramnios, prior classical, cesarean delivery or prior myomectomy, placenta previa or placenta accreta, fetal growth restriction, pregestational or gestational diabetes, placental abruption, chorioamnionitis, premature rupture of membranes, cholestasis of pregnancy, alloimmunization of pregnancy and fetal or congenital malformations.

Early-term births (at 37-38 completed weeks) after amniocentesis and confirmation of fetal lung maturity using the standard testing available at each center were the main study group. They were compared with full-term births (at 39-40 week of gestation) associated with a nadir in neonatal risks.

The primary study outcome was a perinatal composite previously associated with early term delivery, defined as one or more of the following: death before hospital discharge, need for respiratory support (ventilator for two or more days, CPAP or surfactant), proven newborn sepsis, pneumonia, meningitis, hypoglycemia requiring treatment, hyperbilirubinemia requiring phototherapy and/or 5 min Apgar < 7. Proven newborn sepsis required positive blood culture results or clinical picture of sepsis syndrome in the setting of infection, pneumonia required $\mathrm{X}$-ray confirmation and meningitis required a culture positive cerebrospinal fluid. Secondary outcomes included the components of the primary composite and specific neonatal respiratory or neonatal morbidities that have either been associated with early delivery or fetal lung maturity testing including respiratory distress syndrome, transient tachypnea of the newborn, cardiopulmonary resuscitation with first 24 hours, bronchopulmonary dysplasia, persistent pulmonary hypertension of the newborn, necrotizing enterocolitis, intraventricular hemorrhage, seizures, NICU admission, hypoxic ischemic encephalopathy and meconium aspiration. The definitions of these outcomes were based on the clinical neonatology diagnoses abstracted from the chart.

Demographic and other characteristics of the study groups were compared using chi-square, Fisher exact, t-tests and Wilcoxon rank tests as appropriate. Similar tests also were used for univariable analyses of outcomes. Prior to multivariable analyses we assessed for effect modification by stratifying the primary outcome results by quality of pregnancy dating (based on trimester of confirmatory ultrasound), prior administration of antenatal steroids, 
labor type and mode of delivery. Logistic regression and also propensity score matching (1:1 and 1:2 matching of early-term with lung maturity testing to full-term on a range of covariates) were used to evaluate the independent relationships in multivariable regression analyses. Propensity scores matching were used as an alternative method to usual logistic regression for estimating the effect of the policy of fetal lung maturity testing from the observational data. Propensity scores is a multivariable analysis methodology that stratifies patients based on probability of receiving an intervention controlling for measured confounding, and may provide a more robust assessment of the relationship between the early delivery after lung maturity testing and outcomes. While 1:1 matching would afford better control for confounding, 1:2 matching would afford more power for the adjustments. The potential confounders (matching factors) considered for both logistic regression and propensity scores analyses respectively included age, parity, BMI, race/ethnicity, mode of delivery, type of labor, smoking, antenatal steroids, quality of dating, neonatal sex, parity and BMI. We did not adjust for type of labor since it is in the intervening pathway and is closely correlated with mode of delivery. Conditional logistic regression models were used in the matched propensity score analysis. SAS version 9.3 was used for the analyses. For all comparisons a p-value $<0.05$ was considered statistically significant; no adjustments were made for multiple comparisons as there was a clearly identified primary outcome of interest.

\section{Results}

A total of 48,137 singleton pregnancies out of the 115,502 pregnancies $(118,422$ babies) in the APEX database met the inclusion criteria for this study (see Figure 1 for flow of inclusion and exclusion criteria). Of note, 365 women who underwent fetal lung maturity testing were among the 40,904 excluded due to medical or obstetric indications; 93 with negative and 272 with positive fetal lung maturity testing at 37-38 weeks. The overall prevalence of fetal lung maturity testing in the absence of medical or obstetric indications for early delivery in the study cohort was $0.52 \%(\mathrm{n}=249)$. There were a total of $180(0.37 \%)$ early term births after positive fetal lung maturity testing. The 47,957 full-term births included 69 who had prior fetal lung maturity testing (42 mature and 27 immature). Of the early term births, $115(63.9 \%)$ were at 37 weeks and $65(37.1 \%)$ at 38 weeks; of full term births, $29070(60.6 \%)$ were at 39 weeks and $18887(39.4 \%)$ at 40 weeks.

The characteristics of the two study groups are presented in Table 1. Parity, BMI and neonatal sex were similar between groups. Mothers of early-term births who had positive fetal lung maturity testing were more likely to be non-Hispanic White, have dates confirmed by first trimester ultrasound or ART, smoke during pregnancy, and have received antenatal steroids.

The results of univariable analyses showing the frequency of each study outcome by group and the unadjusted relative risk relative to full-term births are presented in Table 2. The primary composite outcome ( $16.1 \%$ vs. $5.4 \%)$ was more frequent in the early-term group with fetal lung maturity testing. When examined by completed week of gestation, the primary outcome frequency was $20 \%$ at 37 weeks, $9.2 \%$ at 38 weeks, $5.3 \%$ at 39 weeks and $5.6 \%$ at 40 weeks. Respiratory support with CPAP and hyperbilirubinemia requiring phototherapy were the most frequent components of the primary composite and both were 
more frequent in the early term birth with positive lung maturity group compared with the full-term birth group. Transient tachypnea of the newborn and NICU stay were also more frequent in the early term group (see Table 2). All 8 perinatal deaths (all stillbirths) were in the fullterm group, but no outcome (including perinatal death) was statistically significantly more frequent in the full-term group. Furthermore, none of the 8 stillbirths were born to any of the 69 women who delivered at full term with prior fetal lung maturity testing including 27 with immature results.

In stratified analyses (results not shown), the incidence of the primary composite outcome was higher in the early-term group with positive fetal lung maturity testing regardless of quality of pregnancy dating (based on trimester of confirmatory ultrasound), prior administration of antenatal steroids, labor type and mode of delivery. None of these variables was therefore included in multivariable analyses as an effect modifier. Adjusted results from logistic regression analyses (see Table 3) confirmed the unadjusted findings: the primary composite outcome (aOR 3.2, 95\% CI 2.1-4.8), hyperbilirubinemia requiring phototherapy and NICU admission and stay were all significantly increased with early term birth even after confirmed fetal lung maturity compared with full-term births. Analyses by propensity scores matching also confirmed the increase in the primary composite with early term delivery after confirmed fetal lung maturity: ORs 4.3 (1.8-10.5) for $1: 1$ matching and 3.5 (1.8-6.5) for 1:2 matching. When we restricted the analysis to only those with reliable dating (supported by first or second trimester ultrasound) (aOR 3.1 (2.2-4.4)) or without antenatal corticosteroids (aOR 3.1; 2.2-4.3), the primary outcome results were consistent with those from our main analysis.

We conducted supplementary analyses including an additional 2191 with full-term deliveries that had been excluded due to gestational hypertension, preeclampsia and eclampsia bringing the number of full-term deliveries to 50,148. The primary outcome results remained essentially unchanged overall (aOR $3.1 ; 2.1-4.7$ ) and when stratified by mode of delivery vaginal (aOR 2.7 ; 1.4-5.6) and cesarean (aOR 3.4; 2.0-5.6).

\section{Comment}

We found that compared with full-term $\left(39^{0}-40^{6}\right)$ births, elective early-term $\left(37^{0}-38^{6}\right)$ births (i.e. births in the absence of obvious medical or obstetrical indications for early delivery) are associated with worse respiratory and other neonatal outcomes including hyperbilirubinemia requiring phototherapy. Our results also show that early delivery after fetal lung maturity testing is rare in contemporary obstetric practice. Furthermore, we demonstrate an important increase in other neonatal morbidities and NICU stay.

Our findings are consistent with those of reports suggesting that the practice of fetal lung maturity testing and early delivery if positive does not reduce the risk of adverse neonatal outcomes to the levels seen with delivery $39-40$ weeks. ${ }^{2-5}$ The strength of the association of adverse neonatal outcomes following early-term delivery compared with delivery at 39-40 weeks in our study is also similar to findings from prior reports. In addition, our study differs from the prior studies in that we focused on elective deliveries, excluding any recognized medical or obstetric indications for early delivery. Therefore, our study is unique 
in suggesting that the medical or obstetric indication for early delivery alone is not the major driver of adverse neonatal outcomes with early birth after positive lung maturity testing. The low prevalence overall of fetal lung maturity testing in our cohort confirms observations of decreased utilization of fetal lung maturity testing in recent years. ${ }^{8,11-12}$ Although all 8 deaths (stillbirths) were observed in the full-term group, this is not surprising since fetal lung maturity testing is not done in the setting of stillbirths and because the size of the full-term group was so much larger. Although no stillbirth occurred in women who had prior fetal lung maturity testing, these data should not be interpreted to mean that fetal lung maturity testing would have prevented 8 stillbirths from occurring in the full-term group. Those with negative testing would be expectantly managed, with a risk of stillbirth that is not null. We lacked the power to assess this issue and other data suggest that neonatal, postneonatal and infant mortality may be increased with early term compared with full-term delivery. ${ }^{10}$

The strengths of our study include the following: indications for early delivery were excluded to minimize confounding by indication and the data were derived from a wellcharacterized database populated by certified research data collectors who conducted realtime chart abstractions around the time of delivery. ${ }^{9}$ This provides reassurance regarding the validity of our data. We do acknowledge some study limitations. First, while adequate for the primary composite outcome, the number of elective early-term deliveries is relatively small to fully delineate the association with less frequent outcomes such perinatal death, respiratory distress syndrome and other neonatal morbidities. Second, because fetal lung maturity testing is not performed in the setting of stillbirths, all intrauterine deaths were observed in the full-term group. Furthermore, we do not have information on stillbirths in those who had negative fetal lung maturity testing, and therefore were expectantly managed. We have acknowledged the lack of power to demonstrate any differences in stillbirth between groups. We also did not have information regarding the specific lung maturity test used at each center for analysis; although the tests are expected to perform similarly at the cut-offs used for clinical purposes. ${ }^{1}$ Third, we acknowledge that the alternative to purely elective early-term delivery is expectant management during which preeclampsia and other outcomes that may affect neonatal morbidity may occur and not always elective delivery at $39-40$ weeks. However, the large majority of patients are delivered at 39-40 weeks and our data suggest that their newborns do better even when we included those who developed gestational hypertension and preeclampsia/eclampsia in supplementary analyses. Finally, we do not present any data on maternal outcomes or long-term maternal and infant outcomes. We do not anticipate that early-term delivery will be associated with better maternal outcomes compared with full term delivery; a prior study by our group using another cohort suggested that maternal outcomes may be similar or even be worse with early-term delivery compared to full-term delivery in patients undergoing repeat cesarean. ${ }^{13}$

Overall, our findings suggest that amniocentesis for fetal lung maturity testing should not be undertaken to guide purely elective early-term delivery. One role for lung maturity testing may be in the setting of uncertainty regarding the gestational age. ${ }^{6}$ There is an ongoing debate regarding the proper role for fetal lung maturity testing to guide early delivery when there are ongoing concerns about fetal well-being in the absence of a firm indication for early delivery. ${ }^{6,12}$ An example of this scenario is a patient with persistent perception of reduced fetal movement despite reassuring antenatal testing or one with a prior history of 
fetal demise. Considering that newborns delivered after positive fetal lung maturity testing may be at lower risk of adverse fetal outcomes compared to gestational age-matched cohorts, it is argued fetal lung maturity testing may provide reassurance when the need to deliver is uncertain. ${ }^{1,12}$ Our findings do not specifically address such scenarios and patients with these characteristics were not specifically excluded from our study population. Ideally, a clinical trial in which pregnant women are either randomized to (or offered) fetal lung maturity testing versus the alternative (depending on the scenario) of delivery or expectant management until 39-40 weeks would provide answers regarding the best practice. However, in the absence of such data, our findings support recommendations against using amniocentesis to determine early delivery in the absence of medical or obstetrical indications or concern. ${ }^{6}$

\section{Appendix.}

In addition to the authors, other members of the Eunice Kennedy Shriver National Institute of Child Health and Human Development Maternal-Fetal Medicine Units Network are as follows:

University of Alabama at Birmingham, Birmingham, AL - M. Wallace, A. Northen, J. Grant, C. Colquitt

Northwestern University, Chicago, IL - G. Mallett, M. Ramos-Brinson, A. Roy, L. Stein, P. Campbell, C. Collins, N. Jackson, M. Dinsmoor (NorthShore University HealthSystem), J. Senka (NorthShore University HealthSystem), K. Paychek (NorthShore University HealthSystem), A. Peaceman

Columbia University, New York, NY - M.Talucci, M. Zylfijaj, Z. Reid (Drexel U.), R. Leed (Drexel U.), J. Benson (Christiana H.), S. Forester (Christiana H.), C. Kitto (Christiana H.), S. Davis (St. Peter's UH.), M. Falk (St. Peter's UH.), C. Perez (St. Peter's UH.)

University of Utah Health Sciences Center, Salt Lake City, UT - K. Hill, A. Sowles, J. Postma (LDS Hospital), S. Alexander (LDS Hospital), G.Andersen (LDS Hospital), V. Scott (McKay-Dee), V. Morby (McKay-Dee), K.Jolley (UVRMC), J. Miller (UVRMC), B. Berg (UVRMC)

University of North Carolina at Chapel Hill, Chapel Hill, NC - K. Dorman, J. Mitchell, E. Kaluta, K. Clark (WakeMed), K. Spicer (WakeMed), S. Timlin (Rex), K. Wilson (Rex)

University of Texas Southwestern Medical Center, Dallas, TX - L. Moseley, M. Santillan, J. Price, K. Buentipo, V. Bludau, T. Thomas, L. Fay, C. Melton, J. Kingsbery, R. Benezue

University of Pittsburgh, Pittsburgh, PA- H. Simhan, M. Bickus, D. Fischer, T. Kamon (deceased), D. DeAngelis

Case Western Reserve University-MetroHealth Medical Center, Cleveland, OH - B. Mercer, C. Milluzzi, W. Dalton, T. Dotson, P. McDonald, C. Brezine, A. McGrail 
The Ohio State University, Columbus, OH - C. Latimer, L. Guzzo (St. Ann's), F. Johnson, L. Gerwig (St. Ann's), S. Fyffe, D. Loux (St. Ann's), S. Frantz, D. Cline, S. Wylie, P. Shubert (St. Ann's)

University of Texas Medical Branch, Galveston, TX - J. Moss, A. Salazar, A. Acosta, G. Hankins

Wayne State University, Detroit, MI - N. Hauff, L. Palmer, P. Lockhart, D. Driscoll, L. Wynn, C. Sudz, D. Dengate, C. Girard, S. Field

Brown University, Providence, RI - P. Breault, F. Smith, N. Annunziata, D. Allard, J. Silva, M. Gamage, J. Hunt, J. Tillinghast, N. Corcoran, M. Jimenez

The University of Texas Health Science Center at Houston-Children's Memorial Hermann Hospital, Houston, TX - F. Ortiz, P. Givens, B. Rech, C. Moran, M. Hutchinson, Z. Spears, C. Carreno, B. Heaps, G. Zamora

Oregon Health \& Science University, Portland, OR- J. Seguin, M. Rincon, J. Snyder, C. Farrar, E. Lairson, C. Bonino, W. Smith (Kaiser Permanente), K. Beach (Kaiser Permanente), S. Van Dyke (Kaiser Permanente), S. Butcher (Kaiser Permanente)

The George Washington University Biostatistics Center, Washington, DC - E. Thom, Y. Zhao, P. McGee, V. Momirova, R. Palugod, B. Reamer, M. Larsen

Eunice Kennedy Shriver National Institute of Child Health and Human Development, Bethesda, MD - C. Spong, S. Tolivaisa

MFMU Network Steering Committee Chair (Medical University of South Carolina, Charleston, SC) - J. P. VanDorsten, M.D.

\title{
ACKNOWLEDGEMENTS
}

\begin{abstract}
The authors thank Cynthia Milluzzi, RN, and Joan Moss, RNC, MSN for protocol development and coordination between clinical research centers; Elizabeth Thom, $\mathrm{PhD}$, and Yuan Zhao, MS, for protocol/data management and statistical analysis; and Catherine Y. Spong, MD, and Brian M. Mercer, MD, for protocol development and oversight.

The project described was supported by grants from the Eunice Kennedy Shriver National Institute of Child Health and Human Development (NICHD) (HD21410, HD27869, HD27915, HD27917, HD34116, HD34208, HD36801, HD40500, HD40512, HD40544, HD40545, HD40560, HD40485, HD53097, HD53118) and the National Center for Research Resources (UL1 RR024989; 5UL1 RR025764) and its contents do not necessarily represent the official views of the NICHD, National Center for Research Resources, or the National Institutes of Health.
\end{abstract}

\section{REFERENCES}

1. American College of Obstetricians and Gynecologists. ACOG Practice Bulletin No. 97: Fetal lung maturity. Obstet Gynecol 2008; 112(3):717-26. [PubMed: 18757686]

2. Bates E, Rouse D, Chapman V, Mann ML, Carlo W, Tita ATN. Fetal Lung Maturity Testing Before 39 Weeks and Neonatal Outcomes. Obstet Gynecol 2010;116:1288-1295 [PubMed: 21099593]

3. Kamath BD, Marcotte MP, DeFranco EA. Neonatal morbidity after documented fetal lung maturity in late preterm and early term infants. Am J Obstet Gynecol 2011;204(6):518.e1-8. [PubMed: 21752754] 
4. Fang YM, Guirguis P, Borgida A, Feldman D, Ingardia C, Herson V. Increased neonatal morbidity despite pulmonary maturity for deliveries occurring before 39 weeks. J Matern Fetal Neonatal Med 2013;26(1):79-82. [PubMed: 22963341]

5. Vanderhoeven JP, Peterson SE, Gannon EE, Mayock DE, Gammill HS. Neonatal morbidity occurs despite pulmonary maturity prior to 39 weeks gestation. J Perinatol 2014 34(4):322-5. [PubMed: 24434777]

6. American College of Obstetricians and Gynecologists. ACOG Committee Opinion No. 560: Medically indicated late-preterm and early-term deliveries. Obstet Gynecol 2013;121(4):908-10. [PubMed: 23635709]

7. Yarbrough ML, Grenache DG, Gronowski AM. Fetal lung maturity testing: the end of an era. Biomark Med 2014;8(4):509-15. [PubMed: 24796614]

8. Grenache DG, Wilson AR, Gross GA, Gronowski AM. Clinical and laboratory trends in fetal lung maturity testing. Clin Chim Acta 2010;411(21-22):1746-9. [PubMed: 20670617]

9. Grobman WA, Bailit JL, Rice MM, et al. Can differences in obstetric outcomes be explained by differences in the care provided? The MFMU Network APEX study. Am J Obstet Gynecol 2014;211(2): 147.e1-147.e16. [PubMed: 24631441]

10. Reddy UM, Bettegowda VR, Dias T, Yamada-Kushnir T, Ko CW, Willinger M. Term pregnancy: a period of heterogeneous risk for infant mortality. Obstet Gynecol 2011;117(6):1279-87. [PubMed: 21606738]

11. Luo G, Norwitz ER. Revisiting amniocentesis for fetal lung maturity after 36 weeks' gestation. Rev Obstet Gynecol 2008;1(2):61-8. [PubMed: 18769658]

12. Towers CV, Freeman RK, Nageotte MP, Garite TJ, Lewis DF, Quilligan EJ. The case for amniocentesis for fetal lung maturity in late-preterm and early-term gestations. Am J Obstet Gynecol 2014;210(2):95-6. [PubMed: 24139938]

13. Tita ATN, Lai Y, Landon M et al. Timing of Elective Repeat Cesarean Delivery at Term and Maternal Perioperative Outcomes. Obstet Gynecol 2011;117:280-6. [PubMed: 21252740]

14. Spong CY, Mercer BM, D'alton M, Kilpatrick S, Blackwell S, Saade G. Timing of indicated latepreterm and early-term birth. Obstet Gynecol 2011;118(2 Pt 1):323-33. [PubMed: 21775849] 


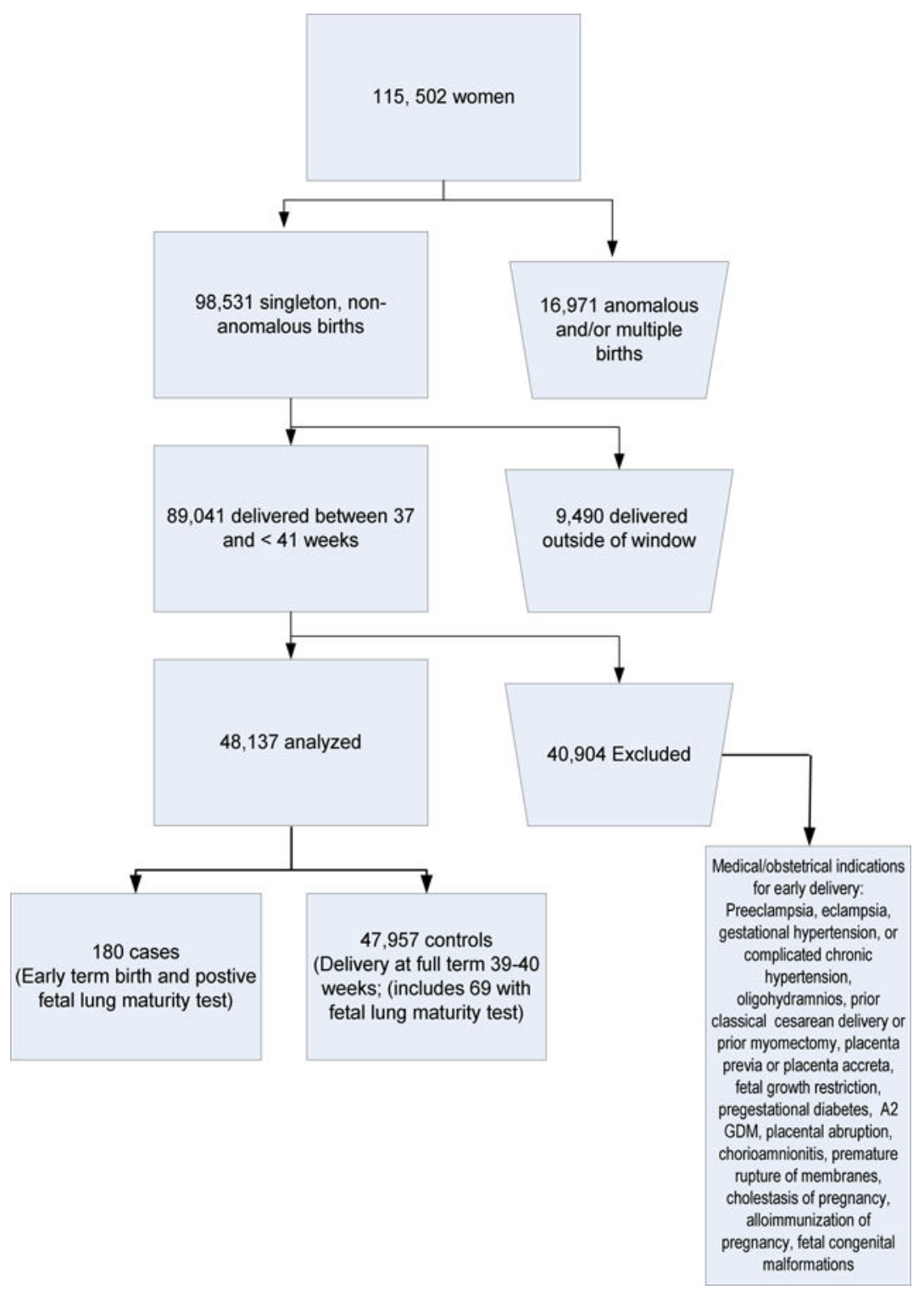

Figure 1: Flow chart of inclusion and exclusion criteria

A total of 48,137 singleton pregnancies out of the 115,502 pregnancies $(118,422$ babies $)$ in the APEX database met the inclusion criteria for this study, most exclusions were due to indications for delivery. 
Table 1.

Baseline Characteristics by Gestational Age at Delivery

\begin{tabular}{|c|c|c|c|}
\hline & Early-term FLM ${ }^{*}$ & Full-term (39-40) & \\
\hline \multirow[t]{2}{*}{ Baseline Characteristic } & & & $P$ val \\
\hline & $\mathrm{N}=180$ & $\mathrm{~N}=47957$ & \\
\hline Age, yrs, median (min to max & $30.0(18.0$ to 56.0$)$ & $28.0(12.0$ to 57.0$)$ & $<0.001$ \\
\hline $\begin{array}{l}\text { BMI, } \mathrm{km} / \mathrm{m}^{2} \text {, median (min to } \\
\max \text { ) }\end{array}$ & $30.6(21.5$ to 61.8$)$ & $29.7(12.4$ to 99.1$)$ & 0.950 \\
\hline Race/ethnicity & & & 0.007 \\
\hline Non-hispanic white & $112(62.2)$ & $23196(48.4)$ & \\
\hline Non-hispanic black & $29(16.1)$ & $8762(18.3)$ & \\
\hline Non-hispanic asian & $5(2.8)$ & $2582(5.4)$ & \\
\hline Hispanic & $30(16.7)$ & $10851(22.6)$ & \\
\hline Other & $3(1.7)$ & $2077(4.3)$ & \\
\hline Not specified & $1(0.6)$ & $489(1.0)$ & \\
\hline Parity, median (min to $\max$ ) & $1.0(0.0$ to 9.0$)$ & $1.0(0.0$ to 11.0$)$ & 0.840 \\
\hline Mode of labor & & & $<0.001$ \\
\hline Induced & $72(40.0)$ & $10351(21.6)$ & \\
\hline Spontaneous/augmented & $6(3.3)$ & $30396(63.4)$ & \\
\hline Cesarean without labor & $102(56.7)$ & $7210(15.0)$ & \\
\hline Mode of delivery & & & $<0.001$ \\
\hline Vaginal & $68(37.8)$ & $35540(74.1)$ & \\
\hline Cesarean delivery & $112(62.2)$ & $12417(25.9)$ & \\
\hline Dating ultrasound trimester & & & $<0.001$ \\
\hline 1st trimester or ART & $111(62.0)$ & $20617(43.0)$ & \\
\hline $2^{\text {nd }}$ or $3^{\text {rd }}$ trimester & $55(30.7)$ & $22294(46.5)$ & \\
\hline None & $13(7.3)$ & $5027(10.5)$ & \\
\hline Baby sex female & $90(50.0)$ & $24085(50.2)$ & 0.953 \\
\hline Smoker & $23(12.8)$ & 4035 ( 8.4) & 0.033 \\
\hline Steroids for fetal lung maturity & $6(3.3)$ & $228(0.5)$ & $<0.001$ \\
\hline
\end{tabular}

Early-term FLM: delivery at 37-38 weeks after positive fetal lung maturity testing 
Table 2.

The Incidence of the Primary Composite and Other Outcomes by Study Group and Un-Adjusted Results

\begin{tabular}{|c|c|c|c|c|}
\hline Outcomes & Early-Term FLM & Full-term & RR $(95 \%$ CI $)$ & $P$ value \\
\hline & $n=180$ & $n=47957$ & & \\
\hline & n $(\%)$ & n $(\%)$ & & \\
\hline Primary composite outcome & $29(16.1)$ & $2594(5.4)$ & $3.0(2.1-4.2)$ & $<0.001$ \\
\hline \multicolumn{5}{|l|}{ Components: } \\
\hline Perinatal death ${ }^{*}$ & $0(0.0)$ & $8(0.02)$ & & \\
\hline $\begin{array}{l}\text { Ventilation within } 24 \text { hours for } \\
\geq 2 \text { days }\end{array}$ & $1(0.6)$ & $57(0.1)$ & $\begin{array}{l}4.7(0.7- \\
35.6)\end{array}$ & 0.125 \\
\hline CPAP use & $7(3.9)$ & $482(1.0)$ & $3.9(1.9-8.0)$ & $<0.001$ \\
\hline Proven newborn sepsis & $0(0.0)$ & $33(0.1)$ & & \\
\hline Pneumonia & $0(0.0)$ & $77(0.2)$ & & \\
\hline Meningitis & $0(0.0)$ & $0(0.0)$ & & \\
\hline Treated hypoglycemia & $1(0.6)$ & $161(0.3)$ & $\begin{array}{c}1.7(0.2- \\
11.8)\end{array}$ & 0.615 \\
\hline $\begin{array}{l}\text { Phototherapy for } \\
\text { hyperbillirubinemia }\end{array}$ & $17(9.4)$ & $1648(3.4)$ & $2.7(1.7-4.3)$ & $<0.001$ \\
\hline Apgar $<7$ at 5 minutes & $2(1.1)$ & $237(0.5)$ & $2.2(0.6-9.0)$ & 0.251 \\
\hline \multicolumn{5}{|l|}{ Secondary Outcomes } \\
\hline Respiratory distress syndrome & $1(0.6)$ & $118(0.3)$ & $\begin{array}{l}2.3(0.3- \\
16.1)\end{array}$ & 0.416 \\
\hline $\begin{array}{l}\text { Transient tachypnea of the } \\
\text { newborn }\end{array}$ & $14(7.8)$ & $679(1.4)$ & $5.5(3.3-9.1)$ & $<0.001$ \\
\hline CPR within first 24 hours & 0 & $19(0.04)$ & & \\
\hline Bronchopulmonary dysplasia & 0 & $1(0.0)$ & & \\
\hline $\begin{array}{l}\text { Persistent pulmonary } \\
\text { hypertension of the newborn }\end{array}$ & 0 & $38(0.08)$ & & \\
\hline Necrotizing enterocolitis & 0 & $5(0.01)$ & & \\
\hline IVH & 0 & $10(0.02)$ & & \\
\hline Seizures & 0 & $37(0.1)$ & & \\
\hline NICU admission & $23(12.8)$ & $2088(4.3)$ & $2.9(2.0-4.3)$ & $<0.001$ \\
\hline NICU stay (range) ${ }^{* *}$ & $4(0-16)$ & $2(0$ to 63$)$ & N.A. & \\
\hline NICU stay >2 days & $14(7.8)$ & $869(1.8)$ & $4.3(2.6-7.1)$ & $<0.001$ \\
\hline $\begin{array}{l}\text { NICU length of stay }>2 \text { (if } \\
\text { admitted to nicu) }\end{array}$ & $14 / 23(60.9)$ & $869 / 2088$ (41.6) & $1.5(1.0-2.0)$ & 0.025 \\
\hline Meconium aspiration & 0 & $65(0.1)$ & & \\
\hline $\begin{array}{l}\text { Hypoxic-Ischemic } \\
\text { Encephalopathy }\end{array}$ & 0 & $61(0.1)$ & & \\
\hline
\end{tabular}


Table 3.

Independent Relationship Between Neonatal Outcomes and Elective Early Term Delivery

\begin{tabular}{|c|c|}
\hline Outcome & AOR $(95 \% \mathrm{CI})^{*}$ \\
\hline Composite Outcome & $3.2(2.1-4.8)$ \\
\hline \multicolumn{2}{|l|}{ Components of Primary Outcome: } \\
\hline Perinatal Death ${ }^{* *}$ & $\mathrm{n} / \mathrm{a}$ \\
\hline Ventilator support $\geq 2$ days within $24 \mathrm{hrs}$ & $4.7(0.7-36)^{\dagger}$ \\
\hline CPAP & $3.9(1.91-8.0)^{\dagger}$ \\
\hline Treated hypoglycemia (requiring NICU) & $1.7(0.2-11.8)^{\dagger}$ \\
\hline Hyperbilirubinemia requiring treatment & $3.0(1.8-5.0)$ \\
\hline Apgar $<7$ at 5 minutes & $2.2(0.6-9.0)^{\dagger}$ \\
\hline Respiratory distress syndrome (RDS) & $2.3(0.3-16)^{\dagger}$ \\
\hline Transient tachypnea of the newborn (TTN) & $5.5(3.3-9.1)$ \\
\hline NICU admission & $2.8(1.8-4.5)$ \\
\hline NICU Stay $>2$ days & $4.3(2.4-7.7)$ \\
\hline \multicolumn{2}{|c|}{ *AOR=Adjusted Odds Ratio (adjusted for ethnicity, age, mode of delivery, smoking, antenatal steroids, quality of dating) } \\
\hline \multicolumn{2}{|c|}{ Unadjusted RR retained due to small numbers (AORS are the results in bold); } \\
\hline
\end{tabular}

